\title{
UPDATE
}

\section{An update on pelvic inflammatory disease}

\section{J D C Ross}

\section{A review of the publications on pelvic inflammatory disease (PID) over the past 18 months reveals a number of common themes. This brief article highlights some relevant papers which may be of interest and summarises their main messages.}

$\mathrm{F}$ or many doctors, even those with many years of clinical experience, the diagnosis of pelvic inflammatory disease (PID) remains problematical. The sensitivity and specificity of a clinical diagnosis hovers around $50 \%^{1-4}$ and the "gold standard" of laparoscopy is expensive, impractical in an outpatient setting, not $100 \%$ sensitive, ${ }^{5}$ and probably subject to interoperator and intraoperator variability. Transvaginal ultrasound is useful in detecting fluid collections within the pelvis, such as a tubo-ovarian abscess or pyosalpinx, but cannot visualise the fallopian tube wall which limits its use in diagnosing less severe disease. In a small study of patients with severe PID, magnetic resonance imaging was found to be superior to transvaginal ultrasound (95\% sensitivity, $89 \%$ specificity $)^{6}$ but cost, lack of access, and limited data preclude its widespread use.

An alternative approach is using "power Doppler" to improve the performance of transvaginal ultrasound. Doppler ultrasound measures blood flow, and power Doppler makes the technique sensitive enough to detect the hyperaemia associated with fallopian tube inflammation. A recent study using power Doppler transvaginal ultrasound reports a positive predictive value of $91 \%$ and negative predictive value of $100 \%$ in a small group of patients, and was of particular value in those with milder salpingitis without pelvic abscess formation which was not detected with "ordinary" transvaginal ultrasound. ${ }^{6}$ Potential limitations include difficulties in differentiating between endometriosis associated masses and PID (which accounted for two false positive cases), and the high level of expertise required to interpret the scans. None the less, the technique shows promise, particularly in patients with mild to moderate PID often seen in an outpatient setting.

Since even the potential for widespread availability of magnetic resonance imaging or power Doppler is some way off, is there anything we can do to improve the diagnosis of PID at present? The PEACH study is a large randomised controlled trial comparing inpatient and outpatient management using cefoxitin and doxycycline and is recruiting over 1500 patients. Although the results of the trial are not yet available a number of useful analyses have been reported from this large cohort of women with mild to moderate PID. These results are likely to be more generalis- able than those from inpatients with severe disease which are reported in most other clinical trials.

Even in this group of women with milder disease, adnexal tenderness was found to be a sensitive marker (96\%) of endometritis. ${ }^{7}$ Unfortunately, the associated specificity of $4 \%$ was less impressive so, although treating all women with adnexal tenderness is unlikely to miss many cases, a lot of women without PID will receive unnecessary antibiotics. Combining lower abdominal tenderness, adnexal tenderness, and cervical motion tenderness reduced the sensitivity to $83 \%$ with a specificity of $22 \%$ - as might be expected adding more criteria improves the specificity but reduces the sensitivity. On multivariate analysis the two factors which best predicted endometritis were a positive bacterial test result (gonorrhoea or chlamydia) and the combination of elevated temperature with a high white cell count. Interestingly, and counterintuitively, an elevated temperature in isolation was negatively correlated with endometritis. One possible explanation for this is that the temperature is more likely to be the result of some other cause, such as viral gastroenteritis, when a high white cell count is absent. Using a logistic regression model to give additional "weight" to the most important clinical criteria did improve the sensitivity and specificity to $62 \%$ and $77 \%$ respectively, but still leaves considerable room for improvement.

Can we predict which women with PID are going to do badly when they initially present? A retrospective study of 322 women found that only older age (over 35) and self reported drug misuse predicted prolonged hospital admission, need for surgery, or readmission. ${ }^{8}$ This is slightly surprising since additional factors, such as chlamydial infection, ${ }^{9}$ delayed presentation, ${ }^{10}$ and severity of disease $^{11} 12$ have been associated with poorer prognosis in other reports and may suggest this study was underpowered and suffered from its retrospective design. Although the use of oral contraceptives has previously been thought to be protective against PID, data from the PEACH study have recently raised doubts over this issue. $^{1314}$

The association between intrauterine contraceptive devices (IUD) and PID has remained controversial. A meta-analysis published last year ${ }^{15}$ reviewed 36 papers and found a relative risk for symptomatic PID of 3.3 (95\% CI 2.1 to 5.3 ) in women with IUDs, although the majority of studies included were case controlled or cross sectional rather than randomised controlled trials. To understand this increased risk, however, there are a number of important factors to consider.

Firstly, most of the excess risk associated with IUDs appears to be limited to the first few weeks 
after insertion, ${ }^{16}{ }^{17}$ reflecting the introduction of bacteria into the upper genital tract during coil fitting. It therefore follows that a major determinant of PID rates associated with IUD use will be the prevalence of Chlamydia trachomatis and Neisseria gonorrhoeae, which differ according to the population studied. Secondly, the comparison groups in many trials are inappropriate. In particular the control (non-IUD user) groups often contain women using other contraceptives, such as the oral contraceptive, which lower the risk of PID. ${ }^{18}{ }^{19}$ Also sexual behaviour, a major determinant of STD risk, is seldom matched or controlled for between IUD users and control groups. Finally, even if the relative risk of PID is higher in IUD users the absolute risk remains very low ${ }^{20}{ }^{21}$ —of the order of one in 1000-and there does not appear to be an increased risk of tubal infertility. ${ }^{22}$

The question then arises, should antibiotic prophylaxis be given to women before IUD insertion? A Cochrane review, updated at the end of last year, suggests not. ${ }^{23}$ The administration of doxycycline $200 \mathrm{mg}$ immediately did not reduce an already low rate of PID following IUD insertion, even in African populations with high background STD rates. A major problem in establishing whether there is a link between IUD insertion and PID is choosing an appropriate control group. Usually the control group are women getting a coil fitted who do not have cervical infection and they are compared to women with cervical infection getting a coil fitted. This is an unfair comparison since we do not know how many of those with cervical infection would develop PID anyway. The correct control group is women with cervical infection without an IUD-only then can the effect of the IUD be assessed. These findings do not reduce the need to screen for STDs in high risk populations and treat accordingly but provide some reassurance as to the safety of IUDs.

In summary, a number of observations can be made. Power Doppler transvaginal ultrasound may provide a useful diagnostic tool for patients with PID but further larger studies are needed. The clinical diagnosis of PID remains little better than tossing a coin and we continue to rely on prevention, through STD screening, and rapid empirical use of effective antibiotics to control the disease. The risk of IUDs causing PID may have been overestimated in the past and a few months after insertion they pose little if any excess risk. The effectiveness of antibiotic prophylaxis in women having an IUD fitted remains unproved.

\section{ACKNOWLEDGEMENTS}

Conflict of interest: none.

\section{REFERENCES}

1 Bevan CD, Johal BJ, Mumtaz G, et al. Clinical, laparoscopic and microbiological findings in acute salpingitis: report on a United Kingdom cohort. Br J Obstet Gynaecol 1995;102:407-14.

2 CDC. CDC guidelines on sexually transmitted diseases. MMWR 1998:47:RRO2.

3 Morcos R, Frost N, Hnat M, et al. Laparoscopic versus clinical diagnosis of acute pelvic inflammatory disease. J Reprod Med 1993;38:53-6.

4 Jacobson L, Westrom L. Objectivized diagnosis of acute pelvic inflammatory disease. Diagnostic and prognostic value of routine laparoscopy. Am J Obstet Gynecol 1969:105:1088-98.

5 Sellors J, Mahony J, Goldsmith C, et al. The accuracy of clinical findings and laparoscopy in pelvic inflammatory disease. Am J Obstet Gynecol and laparoscopy in pelvic

6 Molander P, Sjoberg J, Paavonen J, et al. Transvaginal power Doppler findings in laparoscopically proven acute pelvic inflammatory disease. Ultrasound Obstet Gynecol 2001;17:233-8.

7 Peipert JF, Ness RB, Blume J, et al. Clinical predictors of endometritis in women with symptoms and signs of pelvic inflammatory disease. $\mathrm{Am} \mathrm{J}$ Obstet Gynecol 2001:184:856-63.

8 Jamieson DJ, Duerr A, Macasaet MA, et al. Risk factors for a complicated clinical course among women hospitalized with pelvic inflammatory disease. Infect Dis Obstet Gynecol 2000;8:88-93.

9 Brunham RC, Binns B, Guijon F, et al. Etiology and outcome of acute pelvic inflammatory disease. J Infect Dis 1988;158:510-17

10 Hillis SD, Joesoef R, Marchbanks PA, et al. Delayed care of pelvic inflammatory disease as a risk factor for impaired fertility. Am J Obstet Gynecol 1993;168:1503-9

11 Soper DE, Brockwell NJ, Dalton HP. Microbial etiology of urban emergency department acute salpingitis: treatment with ofloxacin. Am J Obstet Gynecol 1992;167:653-60.

12 Westrom L. Effect of acute pelvic inflammatory disease on fertility. Am J Obstet Gynecol 1975;121:707-13.

13 Ness RB, Soper DE, Holley RL, et al. Hormonal and barrier contraception and risk of upper genital tract disease in the PID Evaluation and Clinical Health (PEACH) study. Am J Obstet Gynecol 2001;185:121-7.

14 Washington AE, Gove S, Schachter J, et al. Oral contraceptives, Washington AE, Gove $S$, Schachter , et al. Oral contraceptives,
Chlamydia trachomatis infection, and pelvic inflammatory disease. A word of caution about protection. JAMA 1985;253:2246-50.

15 Gareen IF, Greenland S, Morgenstern H. Intrauterine devices and pelvic inflammatory disease: meta-analyses of published studies, 1974-1990. Epidemiology 2000;1 1:589-97

16 Farley TM, Rosenberg M, Rowe PJ, et al. Intrauterine devices and pelvic inflammatory disease: an international perspective. Lancet inflammatory disecis

17 Lee NC, Rubin GL, Borucki R. The intrauterine device and pelvic inflammatory disease revisited: new results from the Women's Health Study. Obstet Gynecol 1988;72:1-6.

18 Wolner-Hanssen P. Oral contraceptive use modifies the manifestations of pelvic inflammatory disease. Br J Obstet Gynaecol 1986;93:619-24.

19 Wolner-Hanssen P, Eschenbach DA, Paavonen J, et al. Decreased risk of symptomatic chlamydial pelvic inflammatory disease associated with of symptomatic chlamydial pelvic inflammatory

20 Walsh T, Grimes D, Frezieres R, et al. Randomised controlled trial of prophylactic antibiotics before insertion of intrauterine devices. IUD Study Group. Lancet 1998;351:1005-8.

21 Shih S, Li-Juan Q, Xuan L. Comparative clinical experience with 3 IUDs, TCu 380 Ag, TCu 220C and Mahua ring, in Tianjin, People's Republic of China. Contraception 1984:29:229-39.

22 Hubacher D, Lara-Ricalde R, Taylor DJ, et al. Use of copper intrauterine devices and the risk of tubal infertility among nulligravid women. N Engl J Med 2001;345:561-7.

23 Grimes DA, Schulz KF. Antibiotic prophylaxis for intrauterine contraceptive device insertion. Cochrane Database Syst Rev 2000;(2):CD001327 [computer file]. 